\title{
Conventions tarifaires et accords entre santésuisse et la FMH
}

\author{
A. Haefeli
}

Deutsch erschienen in Nr. 5/2004

Correspondance:

Dr Andreas Haefel

Président du G7

Holzgass 1

CH-5242 Lupfig
L'introduction du TARMED dans le domaine de la LAMal, le $1^{\text {er }}$ janvier 2004, a exigé de nombreuses mesures d'accompagnement. Dès lors, le G7 a mené l'année dernière d'intenses négociations avec santésuisse afin de régler les questions encore en suspens. Les négociations ont eu lieu dans un climat des plus constructifs, à la recherche de solutions aux problèmes. Ainsi, les négociations contractuelles ont non seulement permis aux sociétés cantonales de médecine de calculer les valeurs initiales du point avec santésuisse, mais aussi d'apporter des solutions aux problèmes les plus divers encore à résoudre.

Les problèmes suivants ont dû être résolus avant la date d'introduction:

\section{Convention sur la facturation des presta- tions paramédicales en cabinet médical}

(Physiothérapie, ergothérapie, logopédie, conseils diététiques et conseils en matière de diabète par du personnel spécialisé engagé par le cabinet médical).

Le médecin engageant ce personnel spécialisé dans son cabinet peut l'annoncer à santésuisse à l'aide de la formule prévue à cet effet et est tenu de demander à la FMH le code EAN correspondant.

Les ouvrages tarifaires exhaustifs peuvent être obtenus auprès de santésuisse ou sur l'internet.

Lorsque le médecin fait exécuter à l'assistante médicale uniquement des traitements faisant appel à l'électrothérapie ou à la thermothérapie (chaleur et froid), il les facture par la position 7320 (tarif de physiothérapie) à l'aide de 10 points. Le tableau publié ci-joint renseigne sur les valeurs du point en vigueur dans chaque canton pour les prestations paramédicales. Les valeurs tarifaires du TARMED ne sont pas applicables dans ce cas.

\section{Groupes professionnels en danger}

Le TARMED pourrait occasionner, à l'endroit de certains groupes professionnels spécialisés, de graves problèmes de revenus liés au tarif. Pour d'autres, la facturation pourrait être involontairement et largement excédentaire.
Afin de remédier à cet état de fait, la FMH et santésuisse ont créé une instance pour les médecins en pratique privée chargée d'élaborer des mesures de correction ciblées à l'intention du bureau de neutralité des coûts.

Vous êtes tous instamment invités à mettre à disposition de vos sociétés cantonales et de discipline médicale les chiffres nécessaires aux corrections à entreprendre.

Comme toutes les expériences l'ont déjà montré dans le domaine de l'AA, on peut s'attendre à ce que l'introduction du TARMED ait une influence particulièrement négative sur les instituts de radiologie, allant jusqu'à menacer leur existence.

Des mesures d'urgence pour la radiologie ont pu être mises au point avec santésuisse. Elles entreront en vigueur le $1^{\text {er }}$ février 2004.

\section{Représentants de la FMH dans les groupes d'intervention en matière de neutralité des coûts}

Bureau pour la neutralité des coûts

- Dr Urs Stoffel, président de la Société médicale zurichoise;

- Dr Charles A. Favrod-Coune, président de la Société médicale vaudoise.

\section{Bureau paritaire d'examen préalable}

- Dr Andreas Haefeli, président de la Société médicale argovienne;

- Dr Markus Wili, président de la Société médicale lucernoise;

- Dr Jürg Pellaton, Société médicale zurichoise.

Les représentants bénéficieront des conseils techniques et statistiques d'une équipe de contrôle financier de NewIndex SA. Jacques-Henri Weidmann, chef du Service tarifaire de la FMH, assurera le lien avec l'organisation TARMED Suisse.

Les décideurs, avec les représentants de santésuisse, auront l'occasion de se préparer à leurs tâches à l'aide d'exercices «d'état-major» sur la base de chiffres réels émanant des années 2002 et 2003. 
Tableau 1

Récapitulation actuelle des valeurs du point: état au 18 janvier 2004. / Aktuelle TPW-Übersicht: Stand 18. Januar 2004.

\begin{tabular}{|c|c|c|c|c|c|c|c|c|}
\hline & \multirow[t]{2}{*}{ 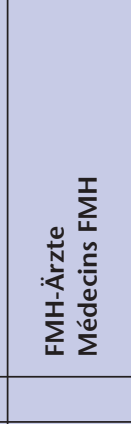 } & \multirow[t]{2}{*}{ 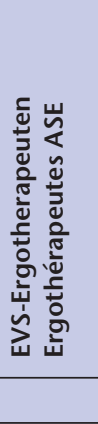 } & \multirow[t]{2}{*}{ 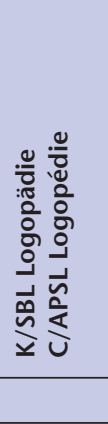 } & \multirow[t]{2}{*}{ 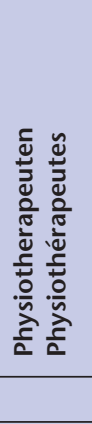 } & \multirow[t]{2}{*}{ 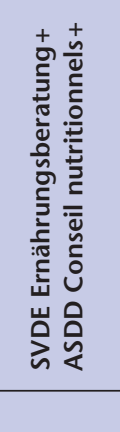 } & \multicolumn{2}{|c|}{ 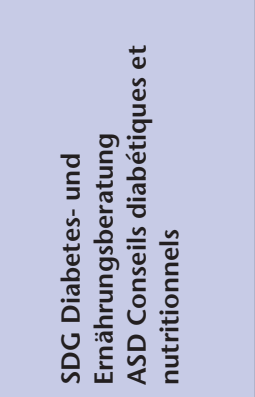 } & \multirow[t]{2}{*}{ 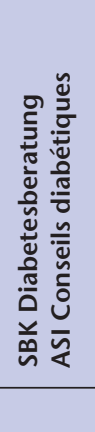 } \\
\hline & & & & & & $D / d$ & $E / n$ & \\
\hline AG & 0,89 & 1,05 & 1,00 & 0,97 & 1,00 & $1,00 \mathrm{TG}$ & $1,00 \mathrm{TG}$ & \multirow{26}{*}{ 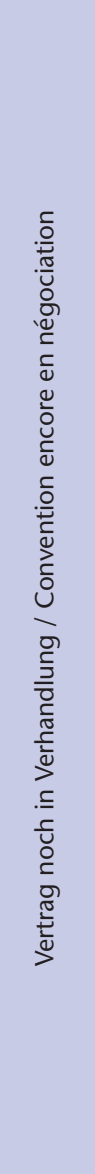 } \\
\hline Al & 0,85 & 1,05 & 1,00 & 0,89 & 1,00 & & & \\
\hline AR & 0,85 & 1,05 & 1,00 & 0,91 & 1,00 & & & \\
\hline $\mathrm{BL}$ & $0,97^{*}$ & 1,05 & 1,00 & 0,95 & 1,00 & $1,00 \mathrm{TG}$ & & \\
\hline BS & $0,93^{*}$ & 1,05 & 1,00 & 0,95 & 1,00 & $1,00 \mathrm{TG}$ & & \\
\hline BE & $0,89^{*}$ & 1,05 & 1,00 & 0,95 & 1,00 & $1,00 \mathrm{TG}$ & $1,00 \mathrm{TG}$ & \\
\hline FR & $0,91^{*}$ & 1,05 & 1,05 & 0,90 & 1,00 & 0,90 TP & 0,90 TP & \\
\hline GE & $0,98^{*}$ & 1,05 & 1,02 & 0,99 & 1,00 & & & \\
\hline GL & 0,85 & $1,05 \mathrm{E}$ & $1,00 \mathrm{E}$ & 0,93 & $1,00 \mathrm{E}$ & & & \\
\hline GR & $0,84^{*}$ & 1,05 & 1,00 & 0,86 & 1,00 & 0,95 TP & & \\
\hline $\mathrm{JU}$ & $0,95^{*}$ & 1,05 & 1,00 & 0,87 & 1,00 & & & \\
\hline LU & $0,86^{*}$ & 1,05 & 1,00 & 0,91 & 1,00 & $1,00 \mathrm{TG}$ & $1,00 \mathrm{TG}$ & \\
\hline $\mathrm{NE}$ & $0,94^{*}$ & 1,05 & 1,00 & 0,88 & 1,00 & & & \\
\hline NW & $0,86^{*}$ & 1,05 & 1,00 & 0,93 & 1,00 & $1,00 \mathrm{TG}$ & 1,00 TG & \\
\hline OW & $0,86^{*}$ & 1,05 & 1,00 & 0,87 & 1,00 & $1,00 \mathrm{TG}$ & $1,00 \mathrm{TG}$ & \\
\hline SG & $0,85^{*}$ & $1,05 \mathrm{E}$ & $1,00 \mathrm{E}$ & 0,90 & $1,00 \mathrm{E}$ & 1,00 TG & & \\
\hline $\mathrm{SH}$ & $0,85^{*}$ & 1,05 & 1,00 & 0,97 & 1,00 & & & \\
\hline$S Z$ & $0,86^{*}$ & 1,05 & 1,00 & 0,91 & 1,00 & $1,00 \mathrm{TG}$ & $1,00 \mathrm{TG}$ & \\
\hline so & $0,87^{*}$ & 1,05 & 1,00 & 0,95 & 1,00 & & & \\
\hline TG & $0,85^{*}$ & $1,05 \mathrm{E}$ & $1,00 \mathrm{E}$ & 0,92 & $1,00 \mathrm{E}$ & $1,00 \mathrm{TG}$ & & \\
\hline $\mathrm{TI}$ & $0,91^{*}$ & 1,05 & 0,95 & 0,87 & $1,00 \mathrm{TG}$ & & & \\
\hline UR & $0,86^{*}$ & 1,05 & 1,00 & 0,90 & 1,00 & $1,00 \mathrm{TG}$ & 1,00 TG & \\
\hline VS & $0,78^{*}$ & 1,05 & 1,00 & 0,88 & 1,00 & 0,85 TP & & \\
\hline VD & $0,92^{*}$ & 1,05 & 1,00 & 0,91 & 1,00 & $0,95 \mathrm{TG}$ & $0,95 \mathrm{TG}$ & \\
\hline ZG & $0,86^{*}$ & 1,05 & $1,00 P$ & 1,03 & 1,00 & $1,00 \mathrm{TG}$ & 1,00 TG & \\
\hline ZH & $0,97^{*}$ & 1,05 & $1,06 \mathrm{E}$ & 1,03 & 1,00 & 1,00 TP & $1,00 \mathrm{TP}$ & \\
\hline \multicolumn{9}{|c|}{ 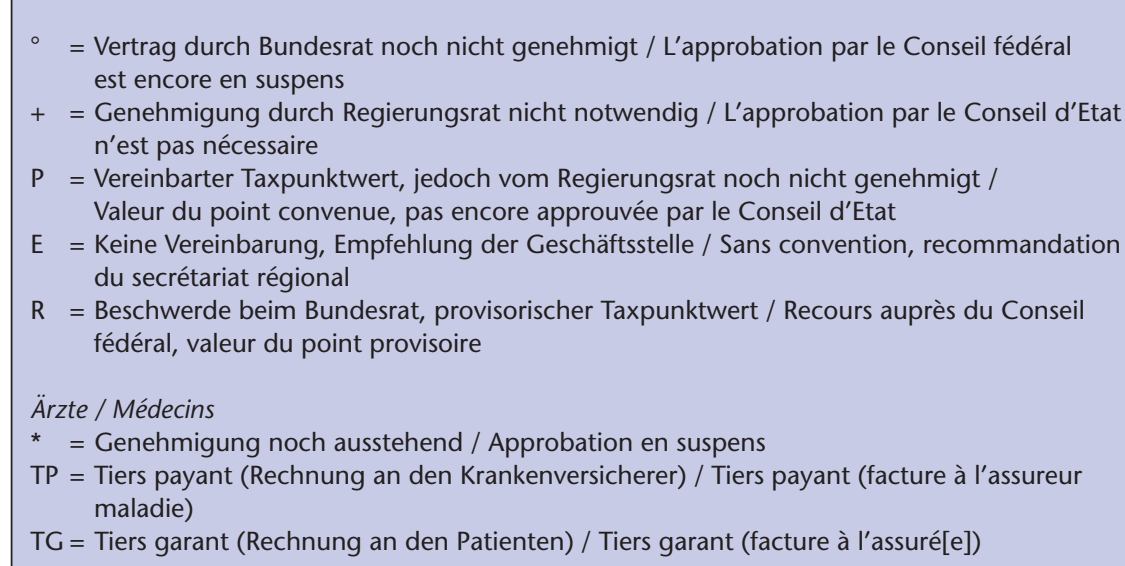 } \\
\hline
\end{tabular}

\section{Facturation}

Les formats des factures TARMED ont pu être adaptés en partenariat à partir des exigences du domaine de l'AA à celui de la LAMal et d'autres lois (p. ex. la loi sur la taxe à la valeur ajoutée). Les documents nécessaires (format de la facture 4.0 et format XML 4.0) ont été mis au point et transmis aux fournisseurs de logiciels.

Vous pouvez commander des formules de facturation manuelle, valables jusqu'à fin juin prochain, auprès de votre société cantonale de médecine.

$\mathrm{Au}$ demeurant, les nombreuses allégations entendues au sujet des données nécessaires à la facturation TARMED sont en grande partie exagérées.

Il s'agit de tenir compte des données impérativement exigées, à savoir celles mentionnées dans la convention-cadre, art. 11, $8^{\mathrm{e}}$ al., ainsi que dans le passage correspondant de votre convention d'adhésion cantonale. Vous vous faciliterez grandement le travail en lisant attentivement ces paragraphes.

\section{Décisions du bureau TARMED pour la neutralité des coûts}

Les décisions du bureau TARMED pour la neutralité des coûts seront publiées la $4^{\mathrm{e}}$ semaine du mois dans le Bulletin des médecins suisses et entreront en vigueur le $1^{\mathrm{er}}$ du mois suivant. 